\title{
PRODUCTS OF COMPOSITION AND DIFFERENTIATION BETWEEN HARDY SPACES
}

\section{ShÛICHI OHNO}

We shall discuss boundedness and compactness of the products of composition and differentiation between Hardy spaces.

\section{INTRODUCTION}

Throughout this article, we denote by $\mathbb{U}$ the open unit disk in the complex plane and by $H^{p}(1 \leqslant p \leqslant \infty)$ the classical Hardy space on $\mathbb{U}$. That is, for $1 \leqslant p<\infty, H^{p}$ is the Banach space of all analytic functions $f$ on $\mathbb{U}$ satisfying

$$
\begin{aligned}
\|f\|_{H^{p}}^{p} & =\sup _{0 \leqslant r<1} \frac{1}{2 \pi} \int_{0}^{2 \pi}\left|f\left(r e^{i \theta}\right)\right|^{p} d \theta \\
& =\lim _{r \rightarrow 1} \frac{1}{2 \pi} \int_{0}^{2 \pi}\left|f\left(r e^{i \theta}\right)\right|^{p} d \theta<\infty
\end{aligned}
$$

and $H^{\infty}$ is the Banach algebra of bounded analytic functions $f$ on $\mathbb{U}$ with the norm

$$
\|f\|_{\infty}=\sup \{|f(z)|: z \in \mathbb{U}\} .
$$

See [4] for more information on the Hardy spaces.

Let $D$ be the differentiation operator and $C_{\varphi}$ the operator of composition with an analytic self-map $\varphi$ of $\mathbb{U}$. Then we define the products of these operators by

$$
C_{\varphi} D f(z)=\left(C_{\varphi} f^{\prime}\right)(z)=f^{\prime}(\varphi(z))
$$

and

$$
D C_{\varphi} f(z)=\left(C_{\varphi} f\right)^{\prime}(z)=f^{\prime}(\varphi(z)) \varphi^{\prime}(z)
$$

for $z \in \mathbb{U}$ and analytic function $f$ on $\mathbb{U}$.

On a general space of analytic functions, $D$ is typically unbounded. On the other hand, it has been showed that $C_{\varphi}$ is bounded on various spaces of analytic functions on

Received 19th October, 2005

The author is partially supported by Grant-in-Aid for Scientific Research (No.17540169), Japan Society for the Promotion of Science.

The author would like to thank the referee for the suggestions for improving the paper more explicitly.

Copyright Clearance Centre, Inc. Serial-fee code: 0004-9727/06 \$A2.00+0.00. 
U $([3,7,9])$, though the products $C_{\varphi} D$ and $D C_{\varphi}$ are possibly still unbounded there. Hibschweiler and Portnoy [5] defined the products $D C_{\varphi}$ and $C_{\varphi} D$ and investigated the boundedness and the compactness of $D C_{\varphi}$ and $C_{\varphi} D$ between weighted Bergman spaces using the Carleson-type measures. But such weighted Bergman spaces would not include the Hardy space case in the characterisation of $C_{\varphi} D$ and so the investigation of boundedness and compactness of $C_{\varphi} D$ between Hardy spaces would remain open. In this article we shall study this problem. That is, in the next section we give the necessary and sufficient conditions for $C_{\varphi} D$ to be bounded and compact between Hardy spaces using the Carleson-type conditions. Moreover, in Section 3, we focus the Hilbert Hardy space $H^{2}$ and present explicit conditions and examples of $C_{\varphi} D$ that is bounded and compact on $H^{2}$. And we also consider when $C_{\varphi} D$ is a Hilbert-Schmidt operator on $H^{2}$.

\section{BetweEn HaRdy SPACES: CARLESON-TYPE CRITERIA}

In this section, we give the necessary and sufficient conditions for $C_{\varphi} D$ to be bounded and compact between Hardy spaces using the Carleson-type conditions. Let $\varphi$ be an analytic self-map of $\mathbb{U}$. We put

$$
\varphi^{*}(\zeta)=\lim _{r \rightarrow 1} \varphi(r \zeta)
$$

for $\zeta \in \partial \mathbb{U}$ whenever this limit exists and associate a measure $\mu$ to $\varphi^{*}$ by setting

$$
\mu(E)=\int_{\left(\varphi^{*}\right)^{-1}(E) \cap \theta U} d \theta / 2 \pi
$$

for $E \subset \overline{\mathbb{U}}$. In other words $\mu$ is the measure on $\overline{\mathbb{U}}$ that satisfies

$$
\int_{\overline{\mathbf{U}}} h d \mu=\int_{\partial \mathrm{U}}\left(h \circ \varphi^{*}\right) d \theta / 2 \pi
$$

for measurable function $h$ on $\overline{\mathbb{U}}$.

Then, for $1 \leqslant p<\infty$ we have

$$
\begin{aligned}
\left\|C_{\varphi} D f\right\|_{H^{p}}^{p} & =\int_{0}^{2 \pi}\left|f^{\prime} \circ \varphi^{*}\left(e^{i \theta}\right)\right|^{p} d \theta / 2 \pi \\
& =\int_{\overline{\mathbf{U}}}\left|f^{\prime}\right|^{p} d \mu .
\end{aligned}
$$

So we can obtain the following equivalences: for $1 \leqslant p, q<\infty, C_{\varphi} D: H^{p} \rightarrow H^{q}$ is bounded (compact, respectively) if and only if the differentiation $D: H^{p} \rightarrow L^{q}(\overline{\mathrm{U}}, d \mu)$ is bounded (compact, respectively).

Here we recall that Luecking [6] and Choe, Koo and Smith [1] characterise the necessary and sufficient conditions for the differentiation $D: H^{p} \rightarrow L^{q}(\mathbb{U}, d \mu)$ to be bounded and compact, for a positive finite Borel measure $\mu$ on $\mathbb{U}$. We extend their results to the measures on $\overline{\mathbb{U}}$. 
For any arc $I$ in $\partial \mathbf{U}$, define the Carleson square over $I$ to be

$$
S_{I}=\left\{r e^{i \theta} \in \overline{\mathrm{U}}: 1-|I| \leqslant r \leqslant 1, e^{i \theta} \in I\right\}
$$

where $|I|$ is $1 / 2 \pi$ times the Euclidean length of $I$.

Then we have the following Carleson-type criteria, which are the so-called "big-oh" and "little-oh" conditions.

THEOREM 2.1. Let $\varphi$ be an analytic self-map of $\mathbb{U}$ and $\mu$ be defined as above. Suppose that $1 \leqslant p<q<\infty$ or $2 \leqslant p=q<\infty$. Then the following hold.

(i) $C_{\varphi} D: H^{p} \rightarrow H^{q}$ is bounded if and only if

$$
\mu\left(S_{I}\right)=O\left(|I|^{q(1+p) / p}\right), \quad I \subset \partial \mathbf{U} .
$$

(ii) $C_{\varphi} D: H^{p} \rightarrow H^{q}$ is compact if and only if

$$
\mu\left(S_{I}\right)=o\left(|I|^{q(1+p) / p}\right), \quad|I| \rightarrow 0 .
$$

Proof: At first suppose that it is satisfied that

$$
\mu\left(S_{I}\right)=O\left(|I|^{q(1+p) / p}\right)
$$

for all $I \subset \partial \mathrm{U}$. Then we can show by the similar method as in the proof of $[2$, Theorem $2.8]$ that $\left.\mu\right|_{\partial v}=0$. So we apply [6, Theorem 3.1] and obtain the equivalence of (i).

Futhermore we can prove (ii) using the following fact whose proof is an easy modification of that of [3, Proposition 3.11]: for $1 \leqslant p, q<\infty, C_{\varphi} D: H^{p} \rightarrow H^{q}$ is compact if and only if $\left\|C_{\varphi} D f_{n}\right\|_{H^{q}} \rightarrow 0$ for every bounded sequence $\left\{f_{n}\right\}_{n}$ in $H^{p}$ such that $f_{n} \rightarrow 0$ uniformly on every compact subset of $\mathbb{U}$. (Refer to $[1$, Lemma 2.5].)

Here we add a result of the case $q=\infty$.

THEOREM 2.2. Let $1 \leqslant p \leqslant \infty$ and $\varphi$ be an analytic self-map of $\mathbb{U}$. Then the following are equivalent:

(i) $C_{\varphi} D: H^{p} \rightarrow H^{\infty}$ is bounded;

(ii) $C_{\varphi} D: H^{p} \rightarrow H^{\infty}$ is compact;

(iii) $\|\varphi\|_{\infty}<1$.

Proof: We prove only that the condition (i) implies (iii). The other implications (ii) $\Rightarrow$ (i) and (iii) $\Rightarrow$ (ii) are clear.

Suppose that $\|\varphi\|_{\infty}=1$ and $|\varphi(\lambda)|=1$ for some $\lambda \in \partial \mathbb{U}$. Then, for $0<\alpha<1$, let

$$
f(z)=\frac{p}{\alpha \overline{\varphi(\lambda)}(1-\overline{\varphi(\lambda)} z)^{\alpha / p}} .
$$

Then $f \in H^{p}$. 
On the other hand,

$$
\left|C D_{\varphi} f(z)\right|=(1-\overline{\varphi(\lambda)} z)^{-(1+\alpha / p)}
$$

and so $C D_{\varphi} f \notin H^{\infty}$.

Finally in this section we pose a question: Characterise the boundedness and compactness of $C_{\varphi} D: H^{p} \rightarrow H^{q}$ in the case that $1 \leqslant q<p<\infty$.

\section{The Hilbert HaRdy SPACE CASE}

We would like to obtain the function-theoretic characterisation. For the purpose we focus the Hilbert Hardy space $H^{2}$.

Before starting our results, we briefly collect some materials for the Nevanlinna counting function that shall be needed in the sequel (refer to [7]).

The Nevanlinna counting function $N_{\varphi}$ of $\varphi$ is defined by

$$
N_{\varphi}(w)=\sum_{\varphi(z)=w} \log \frac{1}{|z|}, \quad w \in \mathbb{U} \backslash\{\varphi(0)\} .
$$

At an extreme case, $N_{\varphi}(\varphi(0))=\infty$.

Firstly we shall require the change of variable formula for integral means of analytic functions using the Nevanlinna counting function: for $f$ analytic on $\mathbb{U}$,

$$
\|f \circ \varphi\|_{H^{2}}^{2}=|f(\varphi(0))|^{2}+2 \int_{\mathbb{U}}\left|f^{\prime}(w)\right|^{2} N_{\varphi}(w) d A(w)
$$

where $d A$ is the normalised area measure on $\mathbb{U}$.

The Nevanlinna counting function has the sub-averaging property as follows: suppose that $\varphi$ is an analytic self-map of $\mathbb{U}$ with $\varphi(0) \neq 0$. If

$$
0<R<|\varphi(0)| \text {, }
$$

then

$$
N_{\varphi}(0) \leqslant \frac{1}{R^{2}} \int_{R U} N_{\varphi} d A
$$

where $R \mathbb{U}=\{|z|<R\}$.

So we obtain the explicit conditions for $C_{\varphi} D$ to be bounded and compact on the Hardy space $H^{2}$, which also are the so-called "big-oh" and "little-oh" conditions.

THEOREM 3.1. Let $\varphi$ be an analytic self-map of $\mathbb{U}$. Then the following hold.

(i) $C_{\varphi} D$ is bounded on $H^{2}$ if and only if

$$
N_{\varphi}(w)=O\left([\log (1 /|w|)]^{3}\right) \quad(|w| \rightarrow 1) .
$$


(ii) $C_{\varphi} D$ is compact on $H^{2}$ if and only if

$$
N_{\varphi}(w)=o\left([\log (1 /|w|)]^{3}\right) \quad(|w| \rightarrow 1) .
$$

Proof: At first we shall show the case (i).

Suppose that $C_{\varphi} D$ is bounded on $H^{2}$. For $\lambda \in \mathbb{U}$, we take the function

$$
f(z)=\frac{\sqrt{1-|\lambda|^{2}}}{1-\bar{\lambda} z}
$$

Then $f \in H^{2}$ and $\|f\|_{H^{2}}=1$. So, using (3.2), we have

$$
\begin{aligned}
\left\|C_{\varphi} D\right\|^{2} & \geqslant\left\|C_{\varphi} D f\right\|_{H^{2}}^{2}=\left\|f^{\prime} \circ \varphi\right\|_{H^{2}}^{2} \\
& =\left|f^{\prime}(\varphi(0))\right|^{2}+2 \int_{\mathbb{U}}\left|f^{\prime \prime}(w)\right|^{2} N_{\varphi}(w) d A(w) \\
& \geqslant\left.\int_{U} \frac{2 \bar{\lambda}^{2}\left(1-|\lambda|^{2}\right)^{1 / 2}}{(1-\bar{\lambda} w)^{3}}\right|^{2} N_{\varphi}(w) d A(w) \\
& =\int_{U} \frac{4\left(1-|\lambda|^{2}\right)|\lambda|^{4}}{|1-\bar{\lambda} w|^{6}} N_{\varphi}(w) d A(w) .
\end{aligned}
$$

Now substituting $w=\alpha_{\lambda}(u)=(\lambda-u) /(1-\bar{\lambda} u)$,

$$
\begin{aligned}
\left\|C_{\varphi} D\right\|^{2} & \geqslant \int_{\mathrm{U}} \frac{4|\lambda|^{4}|1-\bar{\lambda} u|^{2}}{\left(1-|\lambda|^{2}\right)^{3}} N_{\varphi}\left(\alpha_{\lambda}(u)\right) d A(u) \\
& \geqslant \int_{\mathrm{U} / 2} \frac{4|\lambda|^{4}|1-\bar{\lambda} u|^{2}}{\left(1-|\lambda|^{2}\right)^{3}} N_{\varphi}\left(\alpha_{\lambda}(u)\right) d A(u) .
\end{aligned}
$$

Note that $|1-\bar{\lambda} u| \geqslant 1 / 2$ for $u \in \mathbb{U} / 2$. Using the sub-averaging property (3.3) of the Nevanlinna counting function, we obtain

$$
\left\|C_{\varphi} D\right\|^{2} \geqslant \frac{|\lambda|^{4} N_{\varphi}\left(\alpha_{\lambda}(0)\right)}{\left(1-|\lambda|^{2}\right)^{3}}=\frac{|\lambda|^{4} N_{\varphi}(\lambda)}{\left(1-|\lambda|^{2}\right)^{3}}
$$

for $\lambda \in \mathbb{U} \backslash\{\varphi(0)\}$.

Since $\log (1 /|\lambda|)$ is comparable to $1-|\lambda|$ as $|\lambda| \rightarrow 1^{-}$, we obatin

$$
N_{\varphi}(\lambda)=O\left([\log (1 /|\lambda|)]^{3}\right) \quad(|\lambda| \rightarrow 1) .
$$

We shall see the converse. Suppose that for some $R, 0<R<1$, there is a constant $M$ satisfying

$$
\sup _{R<|w|<1} N_{\varphi}(w) /[\log (1 /|w|)]^{3} \leqslant M
$$


For $f$ analytic on $\mathbb{U}$, we use (3.2) and have

$$
\begin{aligned}
\left\|C_{\varphi} D f\right\|_{H^{2}}^{2} & =\left|f^{\prime}(\varphi(0))\right|^{2}+2 \int_{\mathbf{U}}\left|f^{\prime \prime}(w)\right|^{2} N_{\varphi}(w) d A(w) \\
& =\left|f^{\prime}(\varphi(0))\right|^{2}+2\left(\int_{\mathbf{R U}}+\int_{\mathbf{U} \backslash R \mathbf{U}}\right) .
\end{aligned}
$$

The first and the second terms in the right-hand of the equality above are:

$$
\left|f^{\prime}(\varphi(0))\right|^{2} \leqslant(1-|\varphi(0)|)^{-4}\|f\|_{H^{2}}^{2}
$$

and

$$
\int_{R U}\left|f^{\prime \prime}(w)\right|^{2} N_{\varphi}(w) d A(w) \leqslant \frac{4}{(1-|\varphi(0)|)^{6}}\|f\|_{H^{2}}^{2}
$$

Next we estimate the third one.

$$
\begin{aligned}
\int_{\mathbf{U} \backslash R \mathbb{U}}\left|f^{\prime \prime}(w)\right|^{2} N_{\varphi}(w) d A(w) & \\
& \leqslant \sup _{R<|w|<1} \frac{N_{\varphi(w)}}{[\log (1 /|w|)]^{3}} \int_{\mathbf{U} \backslash \boldsymbol{U}}\left|f^{\prime \prime}(w)\right|^{2}[\log (1 /|w|)]^{3} d A(w) .
\end{aligned}
$$

Here let $f(z)=\sum_{n=0}^{\infty} a_{n} z^{n} \in H^{2}$ with $\|f\|_{H^{2}}^{2}=\sum_{n=0}^{\infty}\left|a_{n}\right|^{2}$. Then

$$
\begin{aligned}
\int_{U \backslash R \mathbf{U}}\left|f^{\prime \prime}(w)\right|^{2}[\log (1 /|w|)]^{3} d A(w) & \\
& \leqslant \sum_{n=2}^{\infty}\left|a_{n}\right|^{2} n^{2}(n-1)^{2} \int_{0}^{1} r^{2(n-2)}\left(\log \frac{1}{r}\right)^{3} 2 r d r \\
& =\sum_{n=2}^{\infty}\left|a_{n}\right|^{2} n^{2}(n-1)^{2} \int_{0}^{1} t^{n-2}\left(\frac{1}{2} \log \frac{1}{t}\right)^{3} d t
\end{aligned}
$$

substituting $t=r^{2}$. And substitute $u=\log (1 / t)$. Then

$$
\int_{0}^{1} t^{n-2}\left(\log \frac{1}{t}\right)^{3} d t=\int_{0}^{\infty} e^{-(n-1) x} u^{3} d u
$$

Further substituting $x=(n-1) u$, we have

$$
\int_{0}^{\infty} e^{-(n-1) u} u^{3} d u=\frac{1}{(n-1)^{4}} \int_{0}^{\infty} e^{-x} x^{3} d x=\frac{\Gamma(4)}{(n-1)^{4}}
$$

So

$$
\begin{aligned}
\int_{U \backslash R U}\left|f^{\prime \prime}(w)\right|^{2} N_{\varphi}(w) d A(w) & \leqslant \sum_{n=2}^{\infty}\left|a_{n}\right|^{2} n^{2}(n-1)^{2} \frac{\Gamma(4)}{8(n-1)^{4}} \\
& \leqslant 3 \sum_{n=2}^{\infty}\left|a_{n}\right|^{2} \leqslant 3 \sum_{n=0}^{\infty}\left|a_{n}\right|^{2} .
\end{aligned}
$$


Consequently we obtain

$$
\left\|C_{\varphi} D f\right\|_{H^{2}}^{2} \leqslant\left(\frac{1}{(1-|\varphi(0)|)^{4}}+\frac{8}{(1-|\varphi(0)|)^{6}}+6 M\right)\|f\|_{H^{2}}^{2} .
$$

That is, $C_{\varphi} D$ is bounded on $H^{2}$.

To show the case (ii), we take test functions

$$
f_{n}(z)=\frac{\sqrt{1-\left|\lambda_{n}\right|^{2}}}{1-\overline{\lambda_{n}} z}
$$

for a sequence $\left\{\lambda_{n}\right\}$ in $\mathbb{U}$ such that $\left|\lambda_{n}\right| \rightarrow 1$ as $n \rightarrow \infty$. Then $f_{n}$ converges weakly to 0 and so we obtain the desired condition. The converse is routine.

REMARK. Recall that the essential norm $\left\|C_{\varphi} D\right\|_{e}$ of the operator $C_{\varphi} D$ is defined to its distance from the space of all compact operators on $H^{2}$. We obtain the upper and lower estimates of $\left\|C_{\varphi} D\right\|_{e}$ :

$$
\limsup _{|w| \rightarrow 1} \frac{N_{\varphi}(w)}{[\log (1 /|w|)]^{3}} \leqslant\left\|C_{\varphi} D\right\|_{e}^{2} \leqslant K \limsup _{|w| \rightarrow 1} \frac{N_{\varphi}(w)}{[\log (1 /|w|)]^{3}}
$$

where $K>0$ is a constant.

In the case that $\varphi$ is univalent on $\mathbb{U}$, we can easily deduce the following corollary.

Corollary 3.2. Let $\varphi$ be a univalent analytic self-map of U. Then the following hold.

(i) $C_{\varphi} D$ is bounded on $H^{2}$ if and only if

$$
\sup _{w \in \mathbb{U}} \frac{1-|w|}{(1-|\varphi(w)|)^{3}}<\infty
$$

(ii) $C_{\varphi} D$ is compact $H^{2}$ if and only if

$$
\lim _{|w| \rightarrow 1} \frac{1-|w|}{(1-|\varphi(w)|)^{3}}=0
$$

ExAmples. We can give explicit examples of $C_{\varphi} D$ that is bounded or compact. For $0<\alpha \leqslant 1 / 3$, let $\varphi_{\alpha}(z)=1-(1-z)^{\alpha}$ or

$$
\varphi_{\alpha}(z)=\frac{\sigma(z)^{\alpha}-1}{\sigma(z)^{\alpha}+1}
$$

where $\sigma(z)=(1+z) /(1-z)$. The latter $\varphi_{\alpha}$ is called the lens map. Then both $\varphi_{\alpha}$ satisfy

$$
1-\left|\varphi_{\alpha}(z)\right|^{2} \approx|1-z|^{\alpha} \text { for } z \text { near } 1 \text {. }
$$

So using Corollary 3.2, we obtain that $C_{\varphi_{\alpha}} D$ is bounded on $H^{2}$ when $0<\alpha \leqslant 1 / 3$ and furthermore compact on $H^{2}$ when $0<\alpha<1 / 3$. 
We also can find other example in Smith's paper [8]. Let $P \subset \bar{U}$ be a polygon with $P \cap \partial \mathbf{U}=\{1\}$ and with angular aperture $\pi / 3$ at $w=1$. Let $\varphi$ be a Riemann map of $\mathbb{U}$ onto the interior of $P$. He showed that for such a polygonal map $\varphi$,

$$
N_{\varphi}(w)=O\left([\log (1 /|w|)]^{3}\right) \text { as }|w| \rightarrow 1
$$

Then $C_{\varphi} D$ is bounded on $H^{2}$.

Furthermore we consider when a product $C_{\varphi} D$ is a Hilbert-Schmidt operator on $H^{2}$.

THEOREM 3.3. Let $\varphi$ be an analytic self-map of $\mathbb{U}$. Then $C_{\varphi} D$ is a HilbertSchmidt operator on $H^{2}$ if and only if

$$
\sup _{0 \leqslant r<1} \frac{1}{2 \pi} \int_{0}^{2 \pi} \frac{1}{\left(1-\left|\varphi\left(r e^{i \theta}\right)\right|^{2}\right)^{3}} d \theta<\infty .
$$

Proof: At first we show the "if"-part. For the orthonormal basis $\left\{z^{n}\right\}$, we have

$$
\begin{aligned}
\sum_{n=0}^{\infty}\left\|C_{\varphi} D z^{n}\right\|_{H^{2}}^{2} & =\sum_{n=1}^{\infty} \sup _{0 \leqslant r<1}\left\{\frac{1}{2 \pi} \int_{0}^{2 \pi}\left|n \varphi\left(r e^{i \theta}\right)^{n-1}\right|^{2} d \theta\right\} \\
& \leqslant \sup _{0 \leqslant r<1}\left\{\frac{1}{2 \pi} \int_{0}^{2 \pi} \sum_{n=0}^{\infty}(n+1)^{2}\left|\varphi\left(r e^{i \theta}\right)\right|^{2 n} d \theta\right\} \\
& \leqslant \sup _{0 \leqslant r<1}\left\{\frac{1}{2 \pi} \int_{0}^{2 \pi} \frac{2}{\left(1-\left|\varphi\left(r e^{i \theta}\right)\right|^{2}\right)^{3}} d \theta\right\} .
\end{aligned}
$$

Thus $\sum_{n=0}^{\infty}\left\|C_{\varphi} D z^{n}\right\|_{H^{2}}^{2}<\infty$ and so $C_{\varphi} D$ is a Hilbert-Schmidt operator on $H^{2}$.

Conversely suppose that $C_{\varphi} D$ is a Hilbert-Schmidt operator on $H^{2}$. For the orthonormal basis $\left\{z^{n}\right\}$, we have

$$
\begin{aligned}
\infty & >\sum_{n=0}^{\infty}\left\|C_{\varphi} D z^{n}\right\|_{H^{2}}^{2} \\
& =\sum_{n=1}^{\infty} \sup _{0 \leqslant r<1}\left\{\frac{1}{2 \pi} \int_{0}^{2 \pi}\left|n \varphi\left(r e^{i \theta}\right)^{n-1}\right|^{2} d \theta\right\} \\
& \geqslant \sup _{0 \leqslant r<1}\left\{\frac{1}{2 \pi} \int_{0}^{2 \pi} \sum_{n=0}^{\infty}(n+1)^{2}\left|\varphi\left(r e^{i \theta}\right)\right|^{2 n} d \theta\right\} \\
& \geqslant \sup _{0 \leqslant r<1}\left\{\frac{1}{2 \pi} \int_{0}^{2 \pi} \frac{1}{\left(1-\left|\varphi\left(r e^{i \theta}\right)\right|^{2}\right)^{3}} d \theta\right\} .
\end{aligned}
$$

So we obtain the desired condition. 


\section{REFERENCES}

[1] B. Choe, H. Koo and W. Smith, 'Composition operators acting on holomorphic Sobolev spaces', Trans. Amer. Math. Soc. 355 (2003), 2829-2855.

[2] M.D. Contreras and A.G. Hernández-Díaz, 'Weighted composition operators between different Hardy spaces', Integral Equations Operator Theory 46 (2003), 165-188.

[3] C.C. Cowen and B.D. MacCluer, Composition operators on spaces of analytic functions (CRC Press, Boca Raton, 1995).

[4] P.L. Duren, Theory of $H^{p}$ spaces (Academic Press, New York, 1970).

[5] R.A. Hibschweiler and N. Portnoy, 'Composition followed by differentiation between Bergman and Hardy spaces', Rocky Mountain J. Math. 35 (2005), 843-855.

[6] D.H. Luecking, 'Forward and reverse Carleson inequalities for functions in Bergman spaces and their derivatives', Amer. J. Math. 107 (1985), 85-111.

[7] J. H. Shapiro, Composition operators and classical function theory (Springer-Verlag, New York, 1993).

[8] W. Smith, 'Composition operators between Bergman and Hardy spaces', Trans. Amer. Math. Soc. 348 (1996), 2331-2348.

[9] K. Zhu, Operator theory in function spaces (Marcel Dekker, New York, 1990).

Nippon Institute of Technology

Miyashiro

Minami-Saitama 345-8501

Japan

e-mail: ohno@nit.ac.jp 